\title{
Ökonomisierung im Gesundheitswesen als organisationsethische Herausforderung
}

\author{
Georg Marckmann
}

Eingegangen: 21. Januar 2021 / Angenommen: 14. Mai 2021 / Online publiziert: 25. Juni 2021

(C) Der/die Autor(en) 2021

Zusammenfassung Der finanzielle Druck auf die Krankenhäuser in Deutschland führt zu einer Ökonomisierung medizinischer Entscheidungen, die die Qualität der Patientenversorgung beeinträchtigt und das Gesundheitspersonal erheblich belastet. Der vorliegende Beitrag untersucht, welche Möglichkeiten ein organisationsethischer Ansatz bietet, den Herausforderungen durch die Ökonomisierung zu begegnen. Ausgewählte empirische Befunde sollen zunächst verdeutlichen, welche Auswirkungen die Ökonomisierung auf die Patientenversorgung und das Personal in den Krankenhäusern hat. Zudem liefern sie erste Hinweise auf mögliche Handlungsspielräume für die Krankenhäuser. Dabei wird deutlich, dass die Ökonomisierung einen organisationsethischen Ansatz erfordert, der die Handlungsspielräume auf der Mesoebene, d.h. auf der Ebene der einzelnen Krankenhäuser nutzt. Der organisationsethische Ansatz wird in seinen Grundzügen vorgestellt und dann im Sinne eines Wertemanagements als Lösungsperspektive für die Herausforderungen der Ökonomisierung konkretisiert. Abschließend entwickelt der Beitrag Perspektiven, wie klinische Ethikkomitees mit organisationsethischer Arbeit den negativen Auswirkungen der Ökonomisierung auf die Patientenversorgung und das Personal im Krankenhaus entgegenwirken können.

Schlüsselwörter Gesundheitswesen · Ökonomisierung · Organisationsethik · Klinische Ethikberatung 


\title{
Economization in health care as a challenge for organizational ethics
}

\begin{abstract}
Definition of the problem The financial pressure on German hospitals results in an economization of medical decisions which compromises the quality of patient care and constitutes a considerable burden for health care personnel. This article investigates the possibilities of an organizational ethical approach to manage the challenges of economization.

Arguments Selected empirical findings illustrate the impact of economization on patient care and on hospital personnel. They also provide first hints on hospitals' possible actions against economizations.

Results The findings show that economization requires an organizational ethical approach which uses the hospital's possibilities for actions on the meso level. The organizational approach is presented in its main features and then specified in the sense of a value management as a possible solution for the challenges of economization. Finally, the article presents perspectives on how clinical ethics committees can work with an organizational approach against the negative implications of the economization on patient care and personnel in the hospital.
\end{abstract}

Keywords Health care system · Economization - Organizational ethics · Clinical ethics consultation

\section{Einleitung}

Der finanzielle Druck auf die Krankenhäuser in Deutschland ist in den letzten Jahren erheblich gestiegen. Wesentlich bedingt ist diese Entwicklung durch die Umstellung auf ein Vergütungssystem mittels Fallpauschalen (Diagnosis Related Groups, DRGs), bei denen das jeweilige Krankenhaus einen festen Geldbetrag pro Patient mit einer bestimmten Diagnose erhält. Die Krankenhäuer haben damit einen ökonomischen Anreiz, Patienten mit möglichst wenig Ressourcen erfolgreich zu behandeln und die Anzahl an Fällen mit lukrativen Diagnosen zu steigern. Entsprechend sanken die Verweildauern in den letzten Jahren, während die Fallzahlen deutlich anstiegen. Der zunehmende finanzielle Druck ist aber nicht allein auf die DRG-basierte Vergütung zurückzuführen, sondern auch - und vielleicht sogar vor allem - Ergebnis zusätzlicher, für die Krankenhäuser ungünstiger gesundheitspolitischer Rahmenbedingungen. Beispielhaft erwähnt seien die zu geringe Anpassung des DRG-Basisfallpreises, eine unzureichende Investitionsfinanzierung durch die Bundesländer, der fehlende, bedarfsorientierte Abbau von Überkapazitäten durch die Krankenhausplanung der Länder und die zögerliche Eliminierung bekannter Fehlanreize im DRGSystem.

Inzwischen mehren sich die empirischen Hinweise, dass sich der resultierende finanzielle Druck negativ auf die Patientenversorgung und das Krankenhauspersonal auswirkt. Medizinische Entscheidungen werden unangemessen durch betriebswirtschaftliche Überlegungen beeinflusst, was zu einer Über-, Unter- und Fehlversorgung von Patienten führt. Diese Entwicklung wird unter dem Begriff „Ökonomisie- 
rung" kritisch diskutiert (vgl. z. B. Maio 2012; Manzeschke 2011), verschiedene Organisationen wie die Bundesärztekammer (Bundesärztekammer 2015), der Deutsche Ethikrat (Deutscher Ethikrat 2016) und die Arbeitsgemeinschaft der Wissenschaftlichen Medizinischen Fachgesellschaften (AWMF) (Nothacker et al. 2019) haben in Stellungnahmen Position zur Ökonomisierung bezogen. Voraussichtlich werden sich die Herausforderungen durch die Ökonomisierung nur durch gemeinsame Anstrengungen der Akteure auf den verschiedenen Ebenen des Gesundheitswesens einigermaßen bewältigen lassen (Marckmann 2019). Da der erhebliche Kostendruck in den Krankenhäusern vor allem auf politisch zu verantwortende Vorgaben zurückzuführen ist, ist vor allem die Gesundheitspolitik auf Bundes- und Länderebene in der Pflicht, durch eine veränderte Gestaltung der systemischen Rahmenbedingungen eine bedarfsgerechte Versorgung der Patienten im Krankenhaus unter angemessenen Arbeitsbedingungen für das Personal zu gewährleisten. Bislang fehlt allerdings eine übergreifende Strategie für die nachhaltige Finanzierung der Krankenhäuser in Deutschland, zudem dürften sich die finanzielle Situation der gesetzlichen Krankenversicherung (GKV) in Deutschland aufgrund teurer medizinischer Innovationen und des demographischen Wandels in den kommenden Jahren eher verschärfen.

Angesichts der sich voraussichtlich nur wenig ändernden finanziellen Rahmenbedingungen werden sich die Krankenhäuser der Frage stellen müssen, welche Handlungsspielräume es auf der Mesoebene, also auf der Ebene der einzelnen Krankenhäuser gibt, um dem zunehmenden ökonomischen Druck angemessener zu begegnen. Da dabei die defizitären Rahmenbedingungen auf der Makroebene nicht eliminiert werden, handelt es sich in gewisser Weise um eine ethische Mangelverwaltung (Marckmann und Maschmann 2017). Angesichts des erheblichen akuten Problemdrucks können die Krankenhäuser nicht auf veränderte gesundheitspolitische Rahmenbedingungen warten, sondern müssen umgehend reagieren. Hier setzt der vorliegende Beitrag an und prüft, welche Möglichkeiten ein organisationsethischer Ansatz bietet, den Herausforderungen durch die Ökonomisierung zu begegnen. Unabhängig davon bleiben die Akteure in den Krankenhäusern aber in der Verantwortung, auf entsprechende Veränderungen auf übergeordneter, d.h. v.a. gesundheitspolitischer Ebene hinzuweisen. Anhand ausgewählter empirischer Befunde verdeutlicht der Beitrag zunächst, welche Auswirkungen die Ökonomisierung auf die Patientenversorgung und das Personal in den Krankenhäusern hat. Diese liefern zudem bereits erste Hinweise auf mögliche Handlungsspielräume für die Krankenhäuser. Erforderlich ist dabei ein organisationsethischer Ansatz, der zunächst in seinen Grundzügen vorgestellt und dann als Lösungsperspektive für die Herausforderungen der Ökonomisierung konkretisiert wird. Abschließend entwickelt der Beitrag Perspektiven für die organisationsethische Arbeit klinischer Ethikkomitees.

\section{Auswirkungen der Ökonomisierung auf die Patientenversorgung im Krankenhaus}

Welche Auswirkungen die Ökonomisierung auf die Versorgung von Patienten hat, ist methodisch nicht einfach zu ermitteln. In jedem Einzelfall müsste bestimmt werden, welche medizinische Entscheidung ohne die ökonomischen Anreize angemessen 
gewesen wäre. Die verfügbare wissenschaftliche Evidenz beruht folglich ganz wesentlich auf Einschätzungen von Akteuren im Gesundheitswesen, die danach befragt wurden, inwieweit medizinische Entscheidungen durch ökonomische Erwägungen beeinflusst werden und welche Auswirkungen dies auf die Behandlung von Patienten hat. Eine umfassende Literaturübersicht ist im Rahmen des vorliegenden Beitrags nicht möglich. Es sollen im Folgenden deshalb einige Studienergebnisse exemplarisch vorgestellt werden, die verdeutlichen, mit welchen Auswirkungen der Ökonomisierung die Akteure im Krankenhaus konfrontiert sind und welche Hinweise auf Handlungsbedarfe und -perspektiven sich daraus ergeben.

Verschiedene Umfragen weisen darauf hin, dass die aktuellen Rahmenbedingungen in den Krankenhäusern zu unangemessenen Leistungseinschränkungen und Leistungsausweitungen führen. Viele Ärzte geben an, aus Kostengründen auf nützliche Leistungen verzichten zu müssen (sog. Rationierung) (z. B. Boldt und Schöllhorn 2008; Strech et al. 2009). Einschränkungen der Versorgung erfolgen dabei vor allem im Bereich von Pflege und Zuwendung, also bei den sog. ,,weichen“ Faktoren der Patientenversorgung, weniger bei Diagnostik und Therapie (Reifferscheid et al. 2014). Dabei belegen empirische Studien, dass eine höhere Arbeitsbelastung von Pflegekräften bspw. aufgrund von Personaleinsparungen und einer schlechteren Ausbildung die Mortalität von Patienten im Krankenhaus erhöht (Aiken et al. 2014). Die Qualität der Arbeitsumgebung (u.a. Zusammenarbeit zwischen Pflegekräften und Ärzten, Unterstützung durch das Krankenhausmanagement, Partizipation bei relevanten Entscheidungsprozessen des Krankenhauses) stellt dabei einen wesentlichen modulierenden Faktor dar (Aiken et al. 2012).

Zugleich geben viele Ärzte an, dass in ihrem Zuständigkeitsbereich überflüssige Leistungen vorkommen, die nicht nur die Gesundheitsausgaben unnötig steigern und Patienten potenziell verunsichern, sondern die den Patienten auch Schaden zufügen können (Fölsch et al. 2016). Diese Überversorgung ist nach Einschätzung der Befragten nicht nur durch die Erzielung zusätzlicher Erlöse motiviert, sondern auch wesentlich durch die Sorge vor Behandlungsfehlern und Druck der Patienten. In einer eigenen qualitativen Studie berichteten Krankenhausärzte emotionalen Stress, Gewissenskonflikte, Willkür bei Rationierungsentscheidungen und Unehrlichkeit gegenüber den Patienten in Folge des zunehmenden Kostendrucks (Strech et al. 2008).

Interessant - und für die weiteren Überlegungen relevant - ist eine weitere sozialempirische Untersuchung, in der Ärzte und Geschäftsführer danach befragt wurden, ob medizinische Entscheidungen über die Aufnahme, Behandlung und Entlassung von Patienten unangemessen durch wirtschaftliche Überlegungen beeinflusst würden (Wehkamp und Naegler 2017). Während die Mehrzahl der befragten Ärzte eine Überversorgung aus wirtschaftlichen Gründen bejahte, verneinte dies die Mehrzahl der Geschäftsführer. Zudem berichteten die Ärzte über ethische Konflikte, Stresssituationen und Frustration durch den betriebswirtschaftlichen Druck. Eine aktuelle Studie bestätigt zunehmende psychische Belastungen von Klinikärzten (Vincent-Hölper et al. 2020). Zu den Ursachen gehören unter anderem Zeitdruck, schlechte Arbeitsorganisation sowie Arbeitsbedingungen und Klinikvorgaben, die es den Ärzten unmöglich machen, ihre Patienten den eigenen Ansprüchen gemäß zu versorgen. Zugleich liefert die Studie Hinweise auf Ressourcen, die die psychische 
Gesundheit der Ärzte stützen können: Möglichkeiten der Fort- und Weiterbildung, soziale Unterstützung durch Führungskräfte und Kollegen sowie Handlungsspielräume und Möglichkeiten zur Mitwirkung bei Veränderungen.

Insgesamt liefert die empirische Literatur vielfältige Hinweise auf spürbare Auswirkungen der Ökonomisierung im Krankenhaus. Ethisch besonders besorgniserregend ist die berichtete Über- und Unterversorgung von Patienten, verbunden mit Unehrlichkeit und willkürlichen Allokationsentscheidungen, da dies nicht nur die betroffenen Patienten potenziell schädigt, sondern zudem auch das unverzichtbare Vertrauen von Patienten in das System der ärztlichen Hilfe unterminiert. Den Untersuchungen zufolge belastet die Ökonomisierung auch das Gesundheitspersonal, neben ethischen Konflikten drohen Frustration und Beeinträchtigungen der psychischen Gesundheit. Dies hat nicht nur negative Auswirkungen auf die Qualität der Patientenversorgung, sondern dürfte auch die wirtschaftliche Leistungsfähigkeit der Krankenhäuser schwächen. Gleichzeitig liefern die Untersuchungen aber auch Hinweise auf Gestaltungsspielräume der Krankenhäuser, die selbst unter schwierigen wirtschaftlichen Rahmenbedingungen realisierbar erscheinen: Verbesserung von Arbeitsorganisation und interprofessioneller Kooperation, ein wertschätzender Umgang miteinander, gute Führungsqualität und Möglichkeiten zur Mitwirkung bei Entscheidungen und Veränderungen dürften die Krankenhäuser nicht allzu viel Geld kosten. Mögliche ethische Lösungsansätze müssen deshalb (auch oder vielleicht sogar vor allem) an der Organisation des Krankenhauses ansetzen.

\section{Organisationsethik im Krankenhaus}

Ethik im Krankenhaus befasst sich traditionell vor allem mit ethischen Herausforderungen in der Arzt-Patienten-Beziehung. Individualethische Fragen stehen meist auch im Mittelpunkt klinischer Ethikberatung. Gerechtigkeitsethische Fragen der gesundheitlichen Chancengleichheit, des gleichen Zugangs zu Gesundheitsleistungen und der fairen Ressourcenverteilung werden vor allem hinsichtlich der Rahmenbedingungen auf der Makro- bzw. Systemebene diskutiert. Da Über-, Unter- und Fehlversorgung immer medizinische Entscheidungen im Einzelfall zugrunde liegen, erscheint es auf den ersten Blick naheliegend, auch hinsichtlich der Ökonomisierung ethische Lösungen auf der Mikroebene zu suchen. Schließlich kann man von den handelnden Akteuren durchaus erwarten, dass sie sich in ihren professionellen Urteilen nicht unangemessen von finanziellen Anreizen bzw. Erfolgserwartungen beeinträchtigen lassen. In diese Richtung zielt beispielsweise der Klinik-Codex der Deutschen Gesellschaft für Innere Medizin (DGIM) und des Berufsverbands der Deutschen Internisten (BDI), der ,dem Ökonomisierungsprozess eine auf ärztlicher Ethik und Werten beruhende Haltung im Arbeitsalltag" entgegenstellen möchte (Schumm-Draeger et al. 2017, S. A2340). Auch ein neuer Eid wird als mögliche Lösungsperspektive diskutiert (Wils und Baumann-Hölzle 2018). ${ }^{1}$ Angesichts der zweifelsohne auch jetzt noch vorhandenen Entscheidungsspielräume im Einzelfall

\footnotetext{
${ }^{1}$ https://www.zeit.de/2015/46/aerzte-medizin-oekonomie-hippokratischer-eid-patienten (zugegriffen:
} 17. Jan. 2021). 
ist diese „moralische Rückenstärkung“ für die handelnden Akteure durchaus angemessen und zu begrüßen. Allein an die moralische Verantwortung des Einzelnen zu appellieren, greift allerdings zu kurz, da die erheblichen Einflüsse institutioneller Rahmenbedingungen auf medizinische Entscheidungen unberücksichtigt bleiben. Treffend hat dies der Unternehmensethiker Karl Homann formuliert: „Moral kann im Alltag nur dann systematisch praktiziert werden, wenn sie - nicht in jedem Einzelfall, aber per Saldo - dem Akteur Vorteile bringt, und es ist die Aufgabe der Ordnungspolitik - und des Managements -, die Handlungsbedingungen so zu gestalten, dass Moral im Wettbewerb nicht systematisch ausgebeutet werden kann beziehungsweise durch systematische Fehlanreize erodiert“ (Homann 2012).

Die individualethischen Bemühungen müssen deshalb durch organisationsethische Ansätze ergänzt werden (Dinges 2010; Wallner 2015). Die Organisationsethik widmet sich den ethischen Fragen auf Ebene der Organisation, in diesem Fall auf der Ebene des jeweiligen Krankenhauses. Betrachtet wird die Organisation als soziales System, in dem verschiedene Akteursgruppen miteinander kooperieren. Organisationsethik reflektiert zentrale Ziele und Werte, die Strukturen und Prozesse sowie das Verhalten der Menschen in der Organisation leiten. Damit möchte sie einen Beitrag zur Weiterentwicklung der Organisation und damit zur Organisationskultur, d. h. zum „Charakter“ der Organisation, leisten. Die Organisationsethik kann auf diese Weise die Rahmenbedingungen (mit)gestalten, unter denen (medizinische) Entscheidungen im Einzelfall getroffen werden. Als Entscheidungsprämissen können diese Rahmenbedingungen weiter differenziert werden (Wallner 2015): Programme (Leitbild, Visionen, Strategien), Strukturen und Prozesse (Aufbau und Ablauf von Entscheidungen), Personen (die insbesondere als Führungskräfte Entscheidungsprämissen gestalten) sowie die Kultur der Organisation bieten Ansatzpunkte für die organisationsethische Arbeit. $\mathrm{Zu}$ berücksichtigen und entsprechend $\mathrm{zu}$ reflektieren sind dabei die vielfältigen Wechselwirkungen zwischen den verschiedenen Ebenen von Personen, Teams und der Organisation (Dinges 2010) sowie die Verantwortlichkeiten und rollengebundenen Zuständigkeiten der beteiligten Akteure. Gefordert sind hierbei nicht nur die Führungskräfte im Krankenhaus, sondern auch das am Patienten tätige Personal: Neben ihren individualethischen Pflichten in der unmittelbaren Patientenversorgung tragen sie zudem auch eine (Mit-)Verantwortung für die Gestaltung der Organisation.

Wie bei der klinischen Ethikberatung geht es bei der Organisationsethik weniger darum, konkrete moralische Vorgaben für Strukturen und Prozesse in der Organisation zu entwickeln, sondern die Selbstreflexion der Organisation hinsichtlich moralisch relevanter Fragen zu unterstützen und damit einen Beitrag zur Organisationsentwicklung zu leisten. Zudem muss die Organisationsethik auch die Umwelten der Organisation mit reflektieren, in diesem Fall die gesundheitspolitischen Rahmenbedingungen des Krankenhauses. Auch wenn Organisationen in vielen Fällen ihre Umwelten nur eingeschränkt direkt verändern können, so bleibt es doch Aufgabe der verantwortlichen Akteure der Organisation, beispielsweise in politischen Diskursen auf erforderliche Veränderungen in den übergeordneten Rahmenbedingungen des Gesundheitssystems hinzuwirken. 
Tab. 1 Normative Vorgaben für das Krankenhaus (nach Marckmann und Maschmann 2014, S. 163; Wiederabdruck mit Genehmigung von Elsevier)

\begin{tabular}{|c|c|c|}
\hline Ethische Kriterien & & Konkretisierung: organisationsethische Anforderungen \\
\hline $\begin{array}{l}\text { Wohlergehen des } \\
\text { Patienten }\end{array}$ & $\Rightarrow$ & $\begin{array}{l}\text { Gesundheitlichen Nutzen für Patienten optimieren; } \\
\text { Evidenz zu Nutzen \& Schadenspotenzialen der Maßnahmen berücksich- } \\
\text { tigen (Indikationsqualität) }\end{array}$ \\
\hline $\begin{array}{l}\text { Schaden für den Pati- } \\
\text { enten }\end{array}$ & $\Rightarrow$ & $\begin{array}{l}\text { Belastungen \& gesundheitliche Risiken durch die Versorgung minimie- } \\
\text { ren }\end{array}$ \\
\hline $\begin{array}{l}\text { Respekt der Patienten- } \\
\text { Autonomie }\end{array}$ & $\Rightarrow$ & $\begin{array}{l}\text { Patienten informieren, ihre Wünsche respektieren; Selbstbestimmung } \\
\text { fördern; Gesundheitskompetenz stärken }\end{array}$ \\
\hline Gerechtigkeit & $\Rightarrow$ & $\begin{array}{l}\text { Gleichen Zugang zur Versorgung gewährleisten; Patienten in vergleich- } \\
\text { baren Situationen gleich behandeln; gesundheitliche Ungleichheiten } \\
\text { ausgleichen; begrenzte Ressourcen nach definierten Verfahren \& Krite- } \\
\text { rien zuteilen }\end{array}$ \\
\hline Effizienz & $\Rightarrow$ & $\begin{array}{l}\text { Ressourcen für das Erreichen eines Behandlungsziels minimieren; Wirt- } \\
\text { schaftlichkeitsreserven in Strukturen \& Prozessen mobilisieren; Arbeit } \\
\text { effizient organisieren }\end{array}$ \\
\hline $\begin{array}{l}\text { Auswirkungen auf das } \\
\text { soziale Umfeld }\end{array}$ & $\Rightarrow$ & Angehörige der Patienten respektvoll behandeln \& unterstützen \\
\hline $\begin{array}{l}\text { Auswirkungen auf das } \\
\text { Gesundheitspersonal }\end{array}$ & $\Rightarrow$ & $\begin{array}{l}\text { Mitarbeiter respektvoll behandeln \& führen; physische \& psychische } \\
\text { Belastungen minimieren; beruflich fördern }\end{array}$ \\
\hline $\begin{array}{l}\text { Faire Entscheidungs- } \\
\text { prozesse }\end{array}$ & $\Rightarrow$ & $\begin{array}{l}\text { Transparente, konsistente Entscheidungsprozesse mit relevanter, evi- } \\
\text { denzbasierter Begründung, Partizipationsmöglichkeiten und Offenheit } \\
\text { für Revision }\end{array}$ \\
\hline
\end{tabular}

\section{Organisationsethische Handlungsperspektiven im Krankenhaus}

Die exemplarisch aufgeführten empirischen Studien geben vielfältige Hinweise darauf, dass unter dem zunehmenden betriebswirtschaftlichen Druck zentrale Werte der Patientenversorgung und des Umgangs miteinander im Krankenhaus beeinträchtigt werden. Hier kann und muss die Organisationsethik ansetzen: Schließlich ist es ihre Aufgabe, zentrale Werte und Ziele im Krankenhaus zu reflektieren und Lösungsperspektiven für identifizierte Konfliktkonstellationen zu erarbeiten. Welche konkreten Handlungsoptionen bieten sich nun für die Krankenhäuser im Hinblick auf die Auswirkungen der Ökonomisierung? Ein erster Schritt kann darin bestehen, die für die Organisation „Krankenhaus“ relevanten Werte zu herauszuarbeiten. Tab. 1 bietet eine Übersicht, welche normativen Vorgaben für die handelnden Akteure im Krankenhaus leitend sein können bzw. sollten. Da es das zentrale Ziel des Krankenhauses ist, Patienten stationär zu behandeln, müssen sich die Vorgaben vor allem auch an den drei klassischen medizinethischen Prinzipien Wohltun, Nichtschaden und Achtung der Autonomie orientieren, die die ethischen Verpflichtungen gegenüber dem einzelnen Patienten definieren (Beauchamp und Childress 2019). Die normativen Anforderungen der Gerechtigkeit und der Effizienz weisen dann über den einzelnen Patienten hinaus, beziehen sich aber noch wesentlich auf die Patientenversorgung. Das ethische Effizienzgebot verdeutlicht, dass es sich bei Ethik und Ökonomie nicht um sich wechselseitig ausschließende Gegenpole handelt: Ethisch wie ökonomisch ist es gleichermaßen geboten, ein bestimmtes (Behandlungs-)Ergebnis mit möglichst wenig Ressourcen zu erzielen. Insofern vermag der Lösungsansatz konzeptionell nur 
wenig überzeugen, der Ökonomie im Krankenhaus mehr Ethik entgegenstellen zu wollen (vgl. hierzu ausführlicher Marckmann und Maschmann 2014).

Ethische Verpflichtungen bestehen aber auch gegenüber den anderen Akteuren im Krankenhaus: $\mathrm{Zu}$ berücksichtigen sind deshalb Auswirkungen auf Personen im sozialen Umfeld des Patienten und auf das Gesundheitspersonal, welche sich wiederum auf das Wohlergehen des Patienten auswirken können. Nicht zuletzt sind faire Entscheidungsprozesse im Krankenhaus zu gewährleisten: Die Mitarbeiter sollten über relevante Entscheidungen der Krankenhausleitung informiert werden, Führungsentscheidungen sollten konsistent und mit einer sachlich angemessenen Begründung getroffen werden. Zudem sollte das Personal an wichtigen strategischen Entscheidungen und Entwicklungen im Krankenhaus mitwirken können. Die hier vorgeschlagenen organisationsethischen Vorgaben stellen ein normatives Rahmengerüst dar, das von den jeweiligen Krankenhäusern ergänzt und konkretisiert werden muss. Das Leitbild eines Krankenhauses, in dem wesentliche Werte für die Organisation bereits formuliert sind, kann anhand des hier vorgeschlagenen Rahmengerüsts geprüft und ggf. überarbeitet werden. Krankenhauspersonal verschiedenerer Berufsgruppen und Hierarchieebenen sollte an dem Prozess beteiligt werden, damit sich die Mitarbeiter besser mit den formulierten Werten identifizieren können.

In einem zweiten Schritt müssen die Krankenhäuser dann aber dafür Sorge tragen, dass die formulierten Werte auch regelhaft im Alltag der Patientenversorgung umgesetzt werden (Marckmann und Maschmann 2014). Nur auf diese Weise kann sichergestellt werden, dass die normativen Vorgaben auch unter einem hohen betriebswirtschaftlichen Druck die Patientenversorgung und das Miteinander im Krankenhaus verlässlich leiten. Im Rahmen eines solchen Wertemanagements sind diejenigen Bereiche des Krankenhauses zu identifizieren, in denen die formulierten Werte nur eingeschränkt realisiert werden. Anschließend ist durch entsprechende Maßnahmen wie Fortbildungen, Zielvorgaben, Klimazirkel oder Anreizsysteme sicherzustellen, dass die identifizierten Schwachstellen behoben werden. Hierfür ist es wichtig herauszuarbeiten, durch welche Entscheidungsprämissen (Programme, Strukturen, Prozesse, Personen, Kulturen) die Fehlanreize versursacht werden. Idealerweise setzen organisationsethische Interventionen an den Entscheidungsprämission an und tragen dabei zur Organisationsentwicklung bei. Alternativ können organisationsethische Fragestellungen auch innerhalb bestehender Entscheidungsprämission bearbeitet werden (Wallner 2015).

Die Herausforderung eines solchen Wertemanagements besteht darin, dass es sich bei den normativen Vorgaben wie Patientenorientierung, Führungsqualität, Mitarbeiterorientierung oder dem verantwortlichen Umgang mit begrenzten Ressourcen um nicht einfach zu objektivierende und zu messende Faktoren handelt. Wiederholte Mitarbeiterbefragungen können hier ein geeignetes Instrument sein um festzustellen, inwieweit die Leitwerte im Alltag des Krankenhauses unter den schwierigen finanziellen Rahmenbedingungen tatsächlich realisiert werden (Rechkemmer 2015). Schließlich kann das Krankenhauspersonal selbst am besten beurteilen, wie gut auf die individuellen Bedürfnisse der Patienten eingegangen wird, wie die Führungsqualität ist oder ob sorgsam mit den Ressourcen des Krankenhauses umgegangen wird. Da die Prozesse im Krankenhaus nur eingeschränkt regel- und standardisierbar sind, spielen die Leitwerte eine besondere Rolle für die Qualität der Patientenver- 
sorgung. Für die Umsetzung der normativen Vorgaben tragen - neben der Leitung des Klinikums - die Führungskräfte auf den verschiedenen Hierarchieebenen des Krankenhauses eine besondere Verantwortung: Verlässliche Werteorientierung auch unter hohem betriebswirtschaftlichen Druck setzt voraus, dass „Ethik“ zu einer Führungsaufgabe wird (Marckmann 2019; Wehkamp 2015). Vieles spricht dafür, dass eine gute Werteorientierung die Resilienz der betreffenden Organisation stärkt, was wiederum ein wesentlicher Faktor für das wirtschaftliche Überleben bzw. den Erfolg des Krankenhauses unter schwierigen gesundheitspolitischen Rahmenbedingungen darstellen kann.

Eine interessante, empirisch meines Wissens noch nicht beantwortete Frage ist, ob sich ein solches Wertemanagement für das Krankenhaus auch finanziell rechnen könnte (Marckmann und Maschmann 2014). Zunächst sind gewisse Investitionen in einen (aktualisierten) Leitbildprozess, Mitarbeiterbefragungen und entsprechende Steuerungsmaßnahmen erforderlich. Dann könnte das Wertemanagement aber die wirtschaftliche Leistungsfähigkeit eines Krankenhauses verbessern. Zum einen steigert die konsequente Berücksichtigung ethischer Verpflichtungen gegenüber dem Patienten die ethische Qualität der Patientenversorgung. Zum anderen können, darauf weisen die empirischen Studien hin, Verbesserungen in Bereichen wie Arbeitsumgebung, interprofessionelle Kooperation und Führungsqualität mit begrenztem finanziellen Aufwand nicht nur die Motivation und die psychische Gesundheit des Krankenhauspersonals stärken, sondern zudem auch die Versorgungsqualität verbessern. Eine höhere Arbeitszufriedenheit der Mitarbeiter und ein gutes Betriebsklima könnte für das Krankenhaus nicht zuletzt ein relevanter Vorteil bei der Gewinnung von qualifiziertem Personal sein. Aus anderen Branchen gibt es tatsächlich auch empirische Hinweise, dass eine werteorientierte Führungskultur sich für Unternehmen betriebswirtschaftlich rechnen kann (Peus et al. 2010). Es erscheint folglich nicht vertretbar, wenn Krankenhäuser aus finanziellen Gründen auf Maßnahmen zur Förderung der Werteorientierung verzichten.

\section{Perspektiven organisationsethischer Beratung durch klinische Ethikkomitees}

Klinische Ethikkomitees (KEKs) können einen Beitrag zur Organisationsethik und damit zur Organisationsentwicklung des Krankenhauses leisten. Im Gegensatz zur ethischen Beratung im Einzelfall, erfordert dies aber in der Regel ein längerfristiges Engagement in enger Kooperation mit der Klinikleitung und den Führungskräften. Ein erster organisationsethischer Ansatzpunkt eines KEK könnte darin bestehen, die Ziele und Werte des Krankenhauses, wie sie z. B. in einem Leitbild bereits formuliert sind, zu reflektieren (vgl. Tab. 1) und gemeinsam mit der Krankenhausleitung und den Mitarbeitern einen Diskurs zu starten, welche Werte durch den zunehmenden wirtschaftlichen Druck im Alltag der Patientenversorgung gefährdet sind. Alternativ kann das KEK auch direkt mit den Mitarbeitern in einen Austausch treten, um zu ermitteln, wo die aktuellen Rahmenbedingungen nicht mehr oder nur eingeschränkt werteorientiertes Handeln erlauben. Das KEK könnte überdies eine Befragung der Mitarbeitenden anregen, um Schwachstellen in der Werteorientierung zu ermitteln. 
Die Befragung selbst wäre dann aber von der Klinikleitung durchzuführen, zudem muss die Bereitschaft der Klinikleitung gegeben sein, mit entsprechenden Steuerungs-Maßnahmen auf die Ergebnisse zu reagieren. Auch diese Entwicklung kann ein KEK beratend unterstützen. Mit Blick auf die möglicherweise unterschiedlichen Problemwahrnehmungen von Krankenhausärzten und Geschäftsführern (Wehkamp und Naegler 2017) erscheint eine sehr behutsame, nicht-konfrontative Kommunikation zwischen KEK bzw. Mitarbeitern und Klinikleitung geboten. Die Initiativen sollten idealerweise von der Überzeugung getragen sein, dass letztlich alle Beteiligten gemeinsam im selben Boot sitzen, das am besten gemeinsam durch die schwere See der aktuellen Krankenhausfinanzierung zu steuern ist. Sofern es im Rahmen dieser Organisationsentwicklung gelingt, die Werteorientierung des Krankenhauses zu stärken und Verbesserungen im Verhalten der Mitarbeiter untereinander und gegenüber Patienten zu erzielen, können daraus möglicherweise auch günstigere Rahmenbedingungen für die Ethikberatung im Einzelfall resultieren.

\section{Methodisches Vorgehen bei organisationsethischer Entscheidungsunterstützung}

In Analogie zu ethischen Fallbesprechungen bei einzelnen Patienten können Ethikkomitees auch bei organisationsethischen Fragestellungen eine Entscheidungsunterstützung anbieten. Tab. 2 bietet eine Übersicht über die einzelnen Schritte einer organisationsethischen Fallanalyse mit Durchführung einer entsprechenden organisationsethischen Intervention. Da effektive Interventionen häufig Veränderungen in den Entscheidungsprämissen der Organisation erfordern, sollten die Klinikleitung und die betroffenen Führungskräfte mit in die Analyse und Entscheidungsfindung einbezogen werden, da sie die für die Durchführung einer organisationsethischen Intervention tragenden Akteure sind.

Bei der Analyse im ersten Schritt ist insbesondere herauszuarbeiten, welche Entscheidungsprämissen der Organisation involviert sind, um die Problemkonstellation organisationsethisch richtig verorten zu können. Im zweiten Schritt ist zu prüfen, welche organisationsethischen Anforderungen im vorliegenden Fall einschlägig sind (vgl. Tab. 1) und als Grundlage für die Bewertung der im folgenden Schritt herausgearbeiteten Handlungsoptionen, d.h. der möglichen organisationsethischen Interventionen, dienen sollen. In der Synthese erfolgt dann eine übergreifende Bewertung der Handlungsoptionen: Welche Intervention ist organisationsethisch insgesamt am

Tab. 2 Methodik einer organisationsethischen Analyse und Intervention

\begin{tabular}{ll}
\hline 1 & Fragestellung und Problemanalyse \\
2 & Relevante organisationsethische Bewertungsmaßstäbe (vgl. Tab. 1) \\
3 & Handlungsoptionen: mögliche organisationsethische Interventionen \\
4 & Organisationsethische Bewertung der verschiedenen Handlungsoptionen \\
5 & $\begin{array}{l}\text { Synthese: übergreifende Bewertung und Abwägung der Handlungsoptionen } \Rightarrow \text { Auswahl der } \\
\text { am besten geeigneten Intervention }\end{array}$ \\
6 & Durchführung der organisationsethischen Intervention \\
7 & Evaluation und ggf. Anpassung der Intervention \\
\hline
\end{tabular}


besten geeignet, um die herausgearbeiteten Probleme anzugehen? Sofern es keine Handlungsoption gibt, die allen organisationsethischen Anforderungen gerecht wird, können folgende Überlegungen hilfreich sein: In der Abwägung sind die Verpflichtungen gegenüber den Patienten stärker zu gewichten, da ihr Wohlergehen die primäre Zielsetzung der Organisation Krankenhaus darstellt. Sofern hierbei Einschränkungen anderer organisationsethischer Anforderungen (bspw. eine erhöhte Arbeitsbelastung des Krankenhauspersonals) unter den gegebenen Rahmenbedingungen unvermeidbar sind, sollte versucht werden, diese Einschränkungen durch geeignete Maßnahmen (z. B. optimierte Arbeitsumgebungen für das Personal) möglichst weit zu kompensieren. Die anschließend durchgeführte organisationsethische Intervention ist nach einem vorab zu definierenden Zeitraum zu evaluieren und ggf. anzupassen. Insbesondere bei Änderungen von Entscheidungsprämissen in der Organisation wird hierfür ein längerer Zeitraum erforderlich sein.

\section{Fazit}

Der erhebliche finanzielle Druck auf die Krankenhäuser führt den verfügbaren empirischen Studien zufolge zu einer Ökonomisierung medizinischer Entscheidungen mit Über-, Unter- und Fehlversorgung von Patienten und einer zunehmenden Belastung des Krankenhauspersonals. Es erscheint wenig aussichtsreich, dieser Entwicklung allein mit Maßnahmen auf individualethischer Ebenen zu begegnen. Erforderlich ist vielmehr - neben dringenden Veränderungen in den politisch verantworteten Rahmenbedingungen auf der Makroebene - ein organisationsethischer Ansatz, der das Krankenhaus als soziales System und damit die Werthaltungen und Verhaltensweisen des Personals in den Blick nimmt. Dabei sind zunächst die für die Organisation leitenden Werte herauszuarbeiten. Anschließend muss im Rahmen eines Wertemanagements systematisch geprüft werden, inwieweit die Leitwerte im Alltag der Patientenversorgung tatsächlich gelebt werden. Defizite in der Werteorientierung sind dann durch entsprechende Maßnahmen zu beheben. Ein Klinisches Ethikkomitee kann in enger Zusammenarbeit diesen Prozess der ethischen Organisationsentwicklung initiieren und begleiten. Eine stärkere Werteorientierung im Krankenhaus kann die negativen Auswirkungen des betriebswirtschaftlichen Drucks auf die Qualität der Patientenversorgung sowie die Motivation und Gesundheit der Mitarbeiter reduzieren. Vieles spricht dafür, dass dies zudem die wirtschaftliche Leistungsfähigkeit des Krankenhauses verbessern kann, sodass sich die Investition in ein Wertemanagement für das Krankenhaus auch finanziell rechnen könnte. Bleibt zu hoffen, dass immer mehr klinische Ethikkomitees es wagen, im Bereich der Organisationsethik aktiv zu werden, und dass sie dabei auf Krankenhausleitungen treffen, die den Mehrwert einer stärkeren Werteorientierung erkennen und in eine entsprechende kooperative Organisationsentwicklung investieren.

Funding Open Access funding enabled and organized by Projekt DEAL.

Open Access Dieser Artikel wird unter der Creative Commons Namensnennung 4.0 International Lizenz veröffentlicht, welche die Nutzung, Vervielfältigung, Bearbeitung, Verbreitung und Wiedergabe in jeglichem Medium und Format erlaubt, sofern Sie den/die ursprünglichen Autor(en) und die Quelle ord- 
nungsgemäß nennen, einen Link zur Creative Commons Lizenz beifügen und angeben, ob Änderungen vorgenommen wurden.

Die in diesem Artikel enthaltenen Bilder und sonstiges Drittmaterial unterliegen ebenfalls der genannten Creative Commons Lizenz, sofern sich aus der Abbildungslegende nichts anderes ergibt. Sofern das betreffende Material nicht unter der genannten Creative Commons Lizenz steht und die betreffende Handlung nicht nach gesetzlichen Vorschriften erlaubt ist, ist für die oben aufgeführten Weiterverwendungen des Materials die Einwilligung des jeweiligen Rechteinhabers einzuholen.

Weitere Details zur Lizenz entnehmen Sie bitte der Lizenzinformation auf http://creativecommons.org/ licenses/by/4.0/deed.de.

\section{Einhaltung ethischer Richtlinien}

Interessenkonflikt G. Marckmann gibt an, dass kein Interessenkonflikt besteht.

Ethische Standards Für diesen Beitrag wurden vom Autor keine Studien an Menschen oder Tieren durchgeführt. Für die aufgeführten Studien gelten die jeweils dort angegebenen ethischen Richtlinien.

\section{Literatur}

Aiken LH, Sermeus W, Van den Heede K, Sloane DM, Busse R, McKee M et al (2012) Patient safety, satisfaction, and quality of hospital care: cross sectional surveys of nurses and patients in 12 countries in Europe and the United States. BMJ 344:e1717

Aiken LH, Sloane DM, Bruyneel L, Van den Heede K, Griffiths P, Busse R et al (2014) Nurse staffing and education and hospital mortality in nine European countries: a retrospective observational study. Lancet 383(9931):1824-1830

Beauchamp TL, Childress JF (2019) Principles of biomedical ethics. Oxford University Press, New York Oxford

Boldt J, Schöllhorn T (2008) Rationierung ist längst Realität. Ergebnisse einer Fragebogenaktion auf deutschen Intensivstationen. Dtsch Arztebl 105(19):A995-997

Bundesärztekammer (2015) Stellungnahme „Medizinische Indikationsstellung und Ökonomisierung“. Dtsch Arztebl 112(18):A836

Deutscher Ethikrat (2016) Patientenwohl als ethischer Maßstab für das Krankenhaus. Stellungnahme. Deutscher Ethikrat, Berlin

Dinges S (2010) Organisationsethik - Ethikberatung in der Organisation Krankenhaus. In: Dörries A, Neitzke G, Simon A, Vollmann J (Hrsg) Klinische Ethikberatung. Ein Praxisbuch für Krankenhäuser und Einrichtungen der Altenpflege. Kohlhammer, Stuttgart, S 142-162

Fölsch UR, Faulbaum F, Hasenfuß G (2016) Mitgliederbefragung zu „Klug entscheiden“: Wie Internisten das Problem von Über- und Unterversorgung werten. Dtsch Arztebl 113(13):A604-A606

Homann K (2012) Innere Qualität als Produktionsfaktor?! In: CGIFOS-Konferenz „Innere Qualität“ Stuttgart-Hohenheim, 13. Okt. 2012 (Vortrag)

Maio G (2012) Ärztliche Hilfe als Geschäftsmodell? Eine Kritik der ökonomischen Überformung der Medizin. Dtsch Arztebl 109(16):A804-807

Manzeschke A (2011) Die effiziente Organisation. Beobachtungen zur Sinn- und Seinskrise des Krankenhauses. Ethik Med 23:271-282

Marckmann G (2019) Ethik als Führungsaufgabe: Perspektiven für einen ethisch vertretbaren Umgang mit dem zunehmenden Kostendruck in den deutschen Krankenhäusern. In: Dieterich A, Braun B, Gerlinger T, Simon M (Hrsg) Geld im Krankenhaus. Eine kritische Bestandsaufnahme des DRGSystems. Springer VS, Wiesbaden, S 201-218

Marckmann G, Maschmann J (2014) Zahlt sich Ethik aus? Notwendigkeit und Perspektiven des Wertemanagements im Krankenhaus. Z Evid Fortbild Qual Gesundhwes 108(2-3):157-165

Marckmann G, Maschmann J (2017) Ökonomisierung: Ethische Mangelverwaltung. Dtsch Arztebl 114(44):2028-2032

Nothacker M, Busse R, Elsner P, Folsch UR, Gogol M, Jungehulsing GJ et al (2019) Medizin und Ökonomie: Maßnahmen für eine wissenschaftlich begründete, patientenzentrierte und ressourcenbewusste Versorgung. Ein Strategiepapier der Arbeitsgemeinschaft der Wissenschaftlichen Medizinischen Fachgesellschaften (AWMF). Dtsch Med Wochenschr 144:990-996 
Peus C, Kerschreiter R, Frey D, Traut-Mattausch E (2010) What is the value? Economic effects of ethicallyoriented leadership. J Psychol 218(4):198-212

Rechkemmer K (2015) Innere Qualität in Einrichtungen des Gesundheitswesens. In: Marckmann G (Hrsg) Praxisbuch Ethik in der Medizin. Medizinisch Wissenschaftliche Verlagsgesellschaft, Berlin, S 245-256

Reifferscheid A, Pomorin N, Wasem J (2014) Umgang mit Mittelknappheit im Krankenhaus: Rationierung und Überversorgung medizinischer Leistungen im Krankenhaus? Lehrstuhl für Medizinmanagement, Universität Duisburg-Essen, Essen

Schumm-Draeger P-M, Kapitza T, Mann K, Fölsch UR, Müller-Wieland D (2017) Ökonomisierung in der Medizin: Rückhalt für ärztliches Handeln (Klinik Codex „Medizin vor Ökonomie“). Dtsch Arztebl 114(49):A2338-2340

Strech D, Börchers K, Freyer D, Neumann A, Wasem J, Marckmann G (2008) Ärztliches Handeln bei Mittelknappheit. Ergebnisse einer qualitativen Interviewstudie. Ethik Med 20(2):94-109

Strech D, Danis M, Löb M, Marckmann G (2009) Ausmaß und Auswirkungen von Rationierung in deutschen Krankenhäusern. Ärztliche Einschätzungen aus einer repräsentativen Umfrage. Dtsch Med Wochenschr 134:1261-1266

Vincent-Hölper S, Stein M, Pohling U, Felsberg R, Bobbert P, Nienhaus A (2020) Arbeitsbelastung im Krankenhaus: Gemeinsam gegen die Ökonomie. Dtsch Arztebl 117(22):A1043-1047

Wallner J (2015) Organisationsethik: Methodische Grundlagen für Einrichtungen im Gesundheitswesen. In: Marckmann G (Hrsg) Praxisbuch Ethik in der Medizin. Medizinisch Wissenschaftliche Verlagsgesellschaft, Berlin, S 233-243

Wehkamp K (2015) Ethik als Führungs- und Management-Aufgabe. In: Marckmann G (Hrsg) Praxisbuch Ethik in der Medizin. Medizinisch Wissenschaftliche Verlagsgesellschaft, Berlin, S 257-264

Wehkamp K-H, Naegler H (2017) Ökonomisierung patientenbezogener Entscheidungen im Krankenhaus. Eine qualitative Studie zu den Wahrnehmungen von Ärzten und Geschäftsführern. Dtsch Arztebl 114(47):797-804

Wils J-P, Baumann-Hölzle R (2018) Eid und Ethos. Auf dem Weg zu einem neuen Gelöbnis für Ärzte und Ärztinnen. Nomos, Baden-Baden 